\title{
Hypothesis
}

\section{Acupuncture: What Underlies Needle Administration?}

\author{
Tao Liu \\ Traditional Chinese Medicine Department, The 2nd Teaching Hospital, Jilin University, 218 Ziqiang Street, \\ Changchun 130041, Jilin Province, P.R. China
}

\begin{abstract}
Acupuncture is an ancient Chinese therapy with its mode of action unclear and efficacy inconclusive. A lack of attention given to the role of psychosocial context presented in clinical provision of acupuncture may mainly account for the current dilemma in acupuncture research. This psychosocial context induces various cognitive and affective processes in the patient while receiving this treatment. On the basis of the analysis of these psychological factors involved in clinical provision of acupuncture and in light of prior studies on the placebo effect, the author hypothesizes that acupuncture works through potentiation and modulation of a highly organized and somatotopic network of endogenous opioids that links expectation, attention and body schema. This hypothesis, which focuses on the contextual factors involved in clinical provision of acupuncture, has immediate clinical and experimental implications and will take the acupuncture debate much further forward.
\end{abstract}

Keywords: acupoint-acupuncture-attention-body schema-expectation-meridian-opioid systems - placebo

\section{Introduction}

Acupuncture has been defined simplistically as the practice of inserting one or more needles into specific sites on the body surface for therapeutic purposes. If acupuncture is thus defined, however, questions will arise as none of the technical components of clinical provision of acupuncture, such as the depth and duration of needling and the method and intensity of needle stimulation, are directly associated with the magnitude of its therapeutic effects. Moreover, acupuncture is not a single historically stable therapy, and there are different 'schools' or 'version' of acupuncture (1). Despite the considerable variation among different 'schools' of acupuncture practice, they all claim to be effective. What remains consistent across different 'schools' of acupuncture is, nevertheless, the characteristic psychosocial context in which needle insertion is administered.

For reprints and all correspondence: Dr Tao Liu, Traditional Chinese Medicine Department, The 2nd Teaching Hospital, Jilin University, 218 Ziqiang Street, Changchun 130041, Jilin Province, P.R. China.

Tel: +86-0431-88766155; E-mail: cctaoliu@ hotmail.com
To better understand acupuncture, we have to turn to its traditional Chinese medicine (TCM) theory origin. Acupuncture is a treatment modality of TCM that developed a different conceptual and theoretical basis to that of modern western medicine $(1,2)$. TCM focuses on the inseparability and unity of the mind and the body, mental and emotional factors being inextricably linked with physical processes in maintaining health and precipitating illness (3). Without this basic consideration, any appropriate approach to investigating the mode of action of acupuncture can by no means be made.

\section{The Psychosocial Context Underlying Clinical Provision of Acupuncture}

The classic Chinese text 'Inner Classic of Huang Di', which is believed to lay the foundations for acupuncture, highlighted two critical principles that are of the same importance in ensuring the effectiveness of acupuncturezhishen and deqi. According to TCM, the acupuncturist, when performing needling procedure, is required to closely attend to the emotional state of the patient. 
This principle is called zhishen in TCM, which aims to 'gaining full control over the patient's spirit'. Although zhishen is so highly valued, at least no less than deqi that is well documented, by TCM as to be a prerequisite for the effectiveness of acupuncture, a lack of attention has been given to the psychological factors involved in clinical provision of acupuncture in both experimental and clinical research on acupuncture. That this principle of zhishen can hardly be found in the substantial amount of acupuncture literature just demonstrates the extent to which the importance of the psychosocial context underlying acupuncture has been overlooked.

Acupuncture is closely surrounded by a characteristic psychosocial context that essentially differentiates acupuncture from any other medical treatment in providing the patient with the unique cognitive and affective experience with this therapy. These cognitive and affective processes induced in the patient while receiving treatment include enhanced expectations and beliefs, appropriate levels of fear and anxiety and highly focused and spatially directed attention.

\section{Elevated Expectations and Beliefs}

Culture can profoundly shape medicine through shaping patients' beliefs (4). Embedded in unique beliefs and rituals and purported to be an ancient and natural remedy, acupuncture is especially good at inducing beliefs and expectations in patients. Theory basis underlying acupuncture is unrelated to modern science (1), this philosophical divergence from modern science in explaining patterns and rhyme of life can always allow patients to have elevated beliefs and expectations on this therapy. Another contributing factor to higher expectations on the effectiveness of acupuncture is that most patients, for example with chronic pain conditions, turn to acupuncture only after failed experience with conventional therapies. In addition, the invasive nature of acupuncture and the sophisticated manner in which it is applied can to a great extent contribute to patients' beliefs and expectations on the effectiveness of acupuncture. Moreover, daily practice of acupuncture treatment features comprehensive and intimate acupuncturist-patient relationshipthe whole course of a particular treatment session is actually that of the mutual interaction between the acupuncturist and the patient working together as partners, which can remarkably lead to the patient's enhanced beliefs and expectations on the effectiveness of acupuncture (5).

Despite the concern that laying emphasis on the importance of the role of psychological factors in the effectiveness of acupuncture will, as believed, fundamentally undermine the scientific basis of acupuncture, what actually occurs in daily practice of acupuncture is undeniable. Clinical experience shows that beliefs and expectations play a fundamental role in the effectiveness of acupuncture and the magnitude of effects of acupuncture is determined by how and to what extent the patient is involved in this psychosocial context: acupuncture seems to be more effective in the patient with stronger beliefs and higher expectations. This observation proved to be true in a recent study of acupuncture on pain that reported that patient's beliefs and expectations (determined by the perceived assignment to real acupuncture or a placebo) had a greater impact on treatment outcomes than did the actual treatment itself (6). What underscores the importance of perceived assignment is beyond any doubt the power of patients' beliefs and expectations. Given the important role of expectations and beliefs in the effectiveness of acupuncture, a prior study has suggested that the patient informed consent process be explained explicitly in any published report of studies assessing the effectiveness of acupuncture, because how patients are informed about the study intervention to a great extent determines the levels of their beliefs and expectations, hence treatment outcome (2).

Patients' positive beliefs and expectations are always taken for granted when they consider taking a therapy, as is the case for acupuncture. Though we never have a patient who is 'forced' to receive acupuncture, in daily practice of acupuncture we do occasionally treat patients who are precarious, or even suspicious, about the effectiveness of acupuncture. For these patients, acupuncture is not only ineffective but also it may cause some unexpected and clinically unexplainable effects (always unexplainable sensations at site of needling or along meridians depending on individual patients) that are always identified as adverse effects. In some patients, these effects can even last for a period of time and cannot be discontinued until the patient has been convinced that acupuncture makes no harm. It appears that patients' beliefs and expectations determine the direction of acupuncture effects-positive beliefs and expectations induce therapeutic effects, and conversely, negative beliefs and expectations generate negative effects.

The key role of beliefs and expectations in the effectiveness of acupuncture is naturally neglected, due to their inherent involvement in clinical provision of acupuncture - acupuncture as a procedural treatment is rarely, if ever, administrated in the absence of patients' benefit expectations. A simple but meaningful question is, nevertheless, what the outcome of needle insertion is when the patient is not made aware that it is being carried out-hidden administration of acupuncture free of any psychological contamination. Imaginably, hidden administration of acupuncture, if ethically possible, can by no means be associated with a therapeutic procedure. Inserting needles into the body, if it occurs elsewhere other than in the setting of clinical provision of acupuncture, can be nothing more than a painful injury to the body. It is the patient's beliefs and expectations 
that transform needle insertion - an otherwise painful injury to the body - into a therapeutic procedure.

\section{Highly Focused and Spatially Directed Attention Ensured by Appropriate Levels of Fear and Anxiety}

Acupuncture is, at any rate, an invasive procedure, and its procedural administration may look far more invasive than what it virtually is, for individuals cannot fail to know from their experience of daily life that invasive needle insertion into the body will cause inevitable pain and most likely blood shedding. In addition, to achieve the desired deqi sensations, needle manipulations will probably cause unbearable unpleasant or painful sensations. This perceived invasiveness of needle insertion, especially when the needle in being manipulated, causes inevitable fear and anxiety in the patient while receiving treatment. It varies little across individual patients that they are subject to unconditional fear and anxiety in the anticipation and experience of needle insertion. However, during clinical provision of acupuncture, patients' fear and anxiety are not always salient but manifest mainly as highly focused attention, because they are greatly obscured by patients' benefit expectations. As it was suggested in a prior study that greater levels of fear and anxiety contributed to heightened somatic focus (7). Due to this invasiveness induced fear and anxiety, any patient, even if having much experience with acupuncture, cannot fail to focus his or her attention exclusively on the local sites where needles are inserted. That is a prerequisite for the effectiveness of acupuncture: needle administration systematically directs the patient's attention to the specific areas (acupoints) selected according to TCM in different medical conditions, and these regularly located acupoints, when stimulated by different needle manipulations, can be predictive of where and what treatment effects will occur.

Fear and anxiety induced by the invasive nature of acupuncture can make great impacts on patients' mind. A wide range of adverse effects occurring before or during needle administration can be attributable to patients' fear and anxiety including, just list a few, severe nausea, actual fainting, severe dizziness, heavy sweating and vomiting (8). It should be acknowledged, however, that effective acupuncture treatment necessitates an appropriate level of fear and anxiety-undue fear and anxiety will most likely lead to various adverse effects rather than therapeutic effects. It is the patient's enhanced beliefs and benefit expectations on acupuncture that remarkably balance the negative aspects of fear and anxiety and prevent them from amounting to an unduly high level. Benefit expectations and beliefs, even in the presence of fear and anxiety, ensure that he or she is enjoying and experiencing treatment happily and comfortably. Thus at the core of the psychosocial context surrounding acupuncture are the patient's enhanced beliefs and expectations. In addition to the intimate acupuncturist-patient relationship, acupuncture just depends on its invasiveness, fearful in appearance but minimum in essence, to achieve a maximum involvement of the patient successfully and necessarily. Acupuncture can only work after these psychological factors as outlined above have been brought into full play. Thus this psychosocial context is definitely an integral part of acupuncture.

\section{A Highly Organized and Somatotopic Network of Endogenous Opioids that Links Expectation, Attention and Body Schema}

Although acupuncture has been extensively investigated both experimentally and clinically (1,9-12), its mode of action is still inconclusive (13-16). A lack of attention given to the role of psychosocial context in the effectiveness of acupuncture may mainly account for the current dilemma in acupuncture research. Though acupuncture has been dismissed as nothing more than placebo (17), it is worth noting that 'to say that a treatment such as acupuncture isn't better than placebo does not mean that it does nothing' (4). A comprehensive look at the association between acupuncture and placebo may shed light on the mode of action of acupuncture.

\section{Renewed Interest in Placebo}

The placebo effect has reemerged in the public and scientific interest recently partly because of growing interests in alternative medicine such as acupuncture (18). Current research on the placebo effect found that 'subjective' constructs such as expectation and value had identifiable physiological bases that can act as modulators of basic perceptual, motor and internal homeostatic processes (19). The accumulating published demonstrations of the placebo effect as a legitimate subjective phenomenon accompanied by measurable physiological changes provided strong refutation of the conjecture that placebo responses reflected nothing more than response bias or publication biases $(20,21)$. The most definite neurobiological evidence for the legitimate existence of the placebo effect came from experimental works reporting that chemically blocking the endogenous opioid system via naloxone can reverse an otherwise clearly demonstrative placebo manifestation (22-24). Perhaps not coincidentally, this same drug has been shown experimentally to block acupuncture analgesia (25-28).

\section{Potential Role of Attentional Mechanisms in Modulating Placebo Analgesia}

Despite the considerable progress made in our understanding of the neurobiological mechanisms of the 
placebo effect, research in this area is still in its infancy and most of our knowledge originates from the field of pain and analgesia $(19,29)$. Placebo analgesia can be due to the expectation of clinical benefit and Pavlovian conditioning with or without opioid mechanisms involved. An emerging idea is that expectation-based placebo effects are opioid-mediated, but conditioned placebo effects may depend on other mechanisms (30). As far as we know that endogenous opioids have a fundamental role in expectation-induced analgesia, little is known about how and when expectations activate endogenous opioids and the factors that moderate the impact of expectations on the placebo responding are poorly understood $(24,29,31-33)$. Though expectations and beliefs are a critical factor underlying placeboinduced analgesia, placebo expectations alone have not always been found to produce placebo effects $(34,35)$. Thus other factors, in the presence of expectations, may be involved in placebo-induced analgesia (7).

In 1996, Montgomery and Kirsch first reported that specific expectancies produced specific placebo responses (36). On the basis of this work, Benedetti and colleagues analyzed in detail the highly spatial-specific placebo responses caused by target-directed analgesia expectations, and reported that a target-directed expectation induced a specific effect only on the part of the body that was the target of the expectation, and most important, this specific effect was mediated by endogenous opioids (37). These findings indicated that expectation activated opioids did not release throughout the brain, thus affecting the entire body, but rather they were confined to specific neuronal networks with a high-order organization, thus producing specific placebo responses. On the basis of these findings, they proposed a highly organized and somatotopic network of endogenous opioids that links expectation, attention and body schema (internal representation of the body). Importantly, consistent with a behavioral study (7), these studies may have implicated that attentional mechanisms play a casual determinant role in potentiating and modulating expectation induced analgesia.

\section{A Plausible Mode of Action of Acupuncture}

Prior studies have established that acupuncture analgesia is mediated by opioid peptides (25-28). But how endogenous opioids are activated and modulated by needle administration is still unclear. Benedetti and colleagues' study has potential for advancing our understanding of the neurobiological mechanism of both the placebo effect and acupuncture, because it adds one more psychological parameter (i.e. spatially directed expectancy) to the placebo effect, which warrants that a cognitive component (spatially directed attention) must be present in placebo response generation (37).
This expectation-attention-body schema model vividly mimics the manner in which acupuncture works: first, the patient's beliefs and expectations, as outlined above, play fundamental role in the effectiveness of acupuncture, and the patient with higher expectations and more involved in the therapeutic context is more likely to achieve better treatment outcomes. Second, clinic provision of acupuncture features highly focused attention spatially directed to the specific sites of the body by the perceived invasiveness of needle insertion. Third, according to TCM, effects of needle administration are believed to occur on specific parts of the body in a spatial pattern defined by meridian system. On the basis of these considerations, it is reasonable to hypothesize that acupuncture works through potentiation and modulation of this highly organized and somatotopic network of endogenous opioids that links expectation, attention and body schema. In other words, needle insertion into specific points of the body systematically determines how and where expectation activated opioids are released within the CNS.

\section{Role of Needle Insertion}

Only in the presence of the psychological context in which needle insertion is administered, can acupuncture be defined as the stimulation of special points on the body by the insertion of fine needles. When this expectation-attention-body schema model is adapted to interpret the neurobiological mechanism of acupuncture, the essential question is what the role of needle administration is. In Benedetti and colleagues' study, a spatially directed expectation induced a specific effect only on the part of the body that was the target of the expectation. In the case of acupuncture, as hypothesized above, this process is extended and further modulated: a spatially directed expectation induces specific effects on the part of the body that is predicated by what needle manipulations are performed in which acupoints according to TCM. In other words, highly focused attention spatially directed to the incoming needling stimuli can regulate and control the topographic link between expectations and endogenous opioids. Needle administration therefore plays dual pivotal role: first, perceived invasiveness of needle insertion necessarily ensures that highly focused attention be spatially directed to the specific points (selected according to TCM) on the body during the anticipation and experience of needle insertion; second, needle manipulations applied in these points produce spatial-specific stimuli that can modulate expectation activated endogenous opioids. That is to say, in the presence of the patient's benefit expectations, technically different needle manipulations applied in different acupoints selectively activate different neural representations of the body via attentional mechanisms, 
thus determining where and what treatment effects will occur as needed in different medical conditions.

\section{Essence of Acupoints and Meridians}

Acupuncture is a meridian-based therapy, and according to TCM, when an acupoint is stimulated, treatment effects tend to occur on the specific parts of the body along a particular meridian that contains this specific acupoint. That is why an acupoint on the foot or shin can be used to treat eye disease (9) or one on the hand affect the perception of pain, which present a dilemma for modern scientists. Thus the most important question that has to be answered in exploring the mode of action of acupuncture has been what the essence of acupoints and meridians is. What makes this question more complex is that there is to date no conclusive evidence for the existence of either meridians or acupoints (15).

Acupoints refer to certain specifically located sites on the body, and according to TCM, these different specific points can be grouped into different categories in terms of their functional properties. Different groups of acupoints of the same type of functional property, which are located regularly on specific parts of the body, are the equivalent of 12 main meridians that respond to 12 major functions or 'organs' of the body according to TCM. The present hypothesis can have important implications for understanding what the essence of meridians and acupoints is. According to the present hypothesis, what underlies different functional properties of acupoints located on different meridians is most likely to be different activation patterns of endogenous opioids modulated by attentional mechanisms. It is therefore reasonable to hypothesize that acupoints and meridians do not comprise any specific tissues, but rather that they, when being stimulated, produce spatially different attention patterns, neural representations of which are selectively associated with different activation patterns of endogenous opioids.

\section{deqi Sensations}

Most importantly, according to TCM, some characteristic needling-induced sensations (manifesting as numbness, heaviness, distention, and soreness, with spreading sensation) perceived by the patient, referred to as deqi in TCM, mark the valid activation of an acupoint and hence ensure the effectiveness of acupuncture. While performing needling procedure, the acupuncturist will not stop manipulating the needle until the patient has confirmed that the desired specific deqi sensations have been achieved. Thus, deqi sensations do not occur unless the patient's attention is exclusively focused on the incoming needling stimuli caused by needle manipulations in a specific acupoint. Importantly, perceived needling-induced sensations-deqi-mark correctly established association between needle stimulation and expectation mechanisms. Knowledge of how acupuncture stimulation proceeds centrally during the state of deqi is therefore the key to elucidating the CNS mechanism of acupuncture. A recent neuroimaging study of acupuncture reported that perceived deqi sensations were related to the activation of cognitive and affective correlates of the brain (38).

\section{Discussion}

\section{Factors that Affect the Effectiveness of Acupuncture}

Though Placebo is surprisingly hard to define precisely, several factors that affect the placebo effect have been identified including nature of administration (39), practitioner qualities (39-41), and placebo reactors $(42,43)$. Not coincidentally, effectiveness of acupuncture is also subject to the influence of these complex factors $(4,44)$, and nowhere can the influence of these factors be more convincingly demonstrated than in acupuncture. It is worth noting that these factors identified actually reflect different perspectives in recognizing the importance of the role of patients' beliefs and expectations in the clinical effectiveness of acupuncture. In terms of nature of administration, acupuncture is an invasive, painful, and ritually and technically sophisticated intervention. Prior studies suggest that invasive, uncomfortable, and painful interventions tended to enhance placebo responding $(18,39)$, and that individuals are more likely to demonstrate a placebo effect when they are feeling anxious than when they are not feeling anxious $(45,46)$. Here, consistent with a prior behavioral study (7), it is suggested that this invasiveness/anxiety-placebo relationship is due to that invasiveness induced fear and anxiety enhances treatment effects through heightening somatic focus (highly focused and spatially directed attention in the case of acupuncture). In regard to practitioner qualities, the acupuncturist plays an active and central role in clinical provision of acupuncture $(1,5)$. The acupuncturist's clinical experience, intimate relationship with the patient, and competence determine how and to what extent the patient is involved in this treatment and if needling procedure is optimally administrated, hence the magnitude of acupuncture effects. In addition, clinical experience shows that not all patients are responsive to acupuncture treatment, and it is still unknown why certain individuals are more susceptible than others to acupuncture.

\section{The Specific and Non-specific Manner in which Acupuncture Works}

Acupuncture demonstrates a neurobiological process of how expectation of benefits can be directed, through 
needling manipulations applied in different acupoints, to different spatial loci of the body. Acupuncture comprises three indispensable integral components: enhanced expectations and beliefs, highly focused and spatially directed attention to specific points on the body and specific needle manipulations applied in these points. Such three components are of the same importance for the therapeutic effectiveness of acupuncture. Without the underlying psychological processes, needling tends to be nothing more than a painful injury to the body. It is needle insertion and its underlying psychosocial context combined that makes acupuncture treatment.

Given the enhanced contextual factors involved in clinical provision of acupuncture, acupuncture can make impacts in non-specific manner without needle insertion applied, so long as the patient is convinced that treatment has been administered (11). Only the contextual factors as outlined above have been brought into full play, however, can acupuncture work in specific mannerthrough needle insertion controlling and regulating the topographic link between enhanced expectations and endogenous opioids via attentional mechanisms. Thus acupuncture can affect neural processes of pain in both specific and non-specific manner. It is worth noting, however, that the specific manner is related to the nonspecific manner, that is, whether or not acupuncture works in specific manner is determined by the extent to which the non-specific contextual factors have been brought into play. This suggestion that acupuncture depends on non-specific contextual factors to make specific effects underpins Paterson and Dieppe's argument that it is not meaningful to split complex interventions such as acupuncture into specific and nonspecific elements (47).

Modern neuroimaging techniques such as functional magnetic resonance imaging and positron emission tomography provide a means to map the neurophysiological correlates of acupuncture. Human neuroimaging data demonstrate that acupuncture elicits a widely distributed network of brain regions, and it remains challenging to interpret these findings precisely and systematically in answering the question of how acupuncture differs from its placebo/sham control $(48,49)$. One recent study explored differences in neural processing during verum, covert sham and overt sham needle stimulation at acupoint LI-4 in pain patients using PET (10). In this neuroimaging study, verum acupuncture and covert sham differed from overt sham in dorsolateral PFC, rostral ACC and the midbrain activation, and verum acupuncture induced greater increases in brain response within ipsilateral insular cortex than covert sham. Thus the authors hypothesized that activity within the insular cortex may represent specific acupuncture effects while activation of the DLPFC, rACC and midbrain (PAG) may be related to benefit expectations. As far as the suggestion that acupuncture differs from placebo responses in inducing the insula activation, it is unlikely that needle stimulation directly induced the insula activation independent of the neural activations in DLPFC, rACC and midbrain (PAG); what is more likely is, according to the present hypothesis, that the specific insula activation is related to the non-specific neural activations - the specific insula activation resulted from needle stimulation acting on expectation mechanisms that demonstrate the non-specific neural activations in DLPFC, rACC and midbrain (PAG). Moreover, the observed activations in brain areas such as PFC, ACC and insula have been demonstrated to be the neural basis of higherorder cognition including attention (50), which, consistent with the present hypothesis, implicates that acupuncture also involve neural processes supporting attention and higher cognition (48). Thus acupuncture may involve both attentional and expectation mechanisms.

\section{Implications for Acupuncture Research}

The essential question concerning the efficacy of acupuncture has been that if acupuncture generates specific effects superior to its placebo control. Painstaking effort made to give answers to this question demonstrates the difficult journey we have experienced in the attempt to elucidate the mode of action of acupuncture. Initially, research attention has been solely focused on needling procedure due to the beliefs that if acupuncture is efficacious it must be specifically due to this technical procedure. Randomized placebo-controlled trials were therefore introduced to assess the specific efficacy of needle insertion on the basis of the biomedical consideration that efficacious therapy is therapy that proves to be superior to its placebo-control in a randomized, controlled trial (51). When the majority of RCTs of acupuncture fails to show effects beyond a placebo response, acupuncture has been dismissed as nothing more than placebo (17). But if acupuncture is a pure placebo and perceived effects of acupuncture are mere a placebo response with needle insertion playing no role at all, we have to face the question of how clinic provision of acupuncture can produce such a powerful placebo effect and why acupuncture is provided in such a sophisticated manner.

How to evaluate the effectiveness of complex nonpharmacological interventions such acupuncture has long been a question in controversy (52). Due to the reason that the exact mode of action of acupuncture is still unclear, no sound research design to date is unquestionably available for assessing the efficacy of acupuncture $(16,47)$. This hypothesis that acupuncture depends on a characteristic psychosocial context to work challenges the use of the double-blind randomized placebo-controlled trials - the gold standard in clinical trial design-in evaluating the effectiveness of acupuncture, due to the 
reason that this research design focuses solely on needling procedure and deliberately overlooks the importance of the underlying psychosocial context $(51,53)$. In an RCT, contextual factors are treated with contempt and tolerated as nuisance that needs to be controlled for (54). Patients, either in real treatment group or in placebo group, are openly informed that they could receive either the active treatment or the placebo with a chance of $50 \%$, instead of that 'you will be given a powerful effective treatment', as is in the setting of real clinical practice. Under such a situation, patients in an RCT tend to be more absorbed in finding out what intervention-real treatment or placebo - they are actually on than eagerly expecting the therapeutic benefits of the treatment being administered as is in real clinical practice. If it is a new drug under study, this situation makes no difference, so long as the two arms are precisely under the same situation. In the case of acupuncture, however, this reduced level of expectations and beliefs, among other factors, can greatly prevent needle insertion from making impacts, because needle insertion can work only after expectation mechanisms have been brought into full play. That is why acupuncture is more effective in daily practice or pragmatic research settings, where expectation mechanisms are more likely to be brought into full play, than in placebo-controlled research settings, where reduced expectation status fails to generate optimal acupuncture effects.

Moreover, given that acupuncture is a complex procedural intervention, extremely strict blinding measures have to be taken in placebo-controlled trials of acupuncture. A typical example is a placebo-controlled study of acupuncture on fibromyalgia (55). This study recruited patients who had not had acupuncture before and therefore did not know what to expect. To ensure the treatment blindness, patients wore a blindfold while receiving treatment and were stop from communicating too much with other patients and their acupuncturist. In such a research setting, which fundamentally differs from daily practice of acupuncture, almost all the vital elements that help construct the psychosocial context underlying acupuncture were eradicated by the application of these blinding measures, thus the role of needle insertion can hardly manifest.

\section{Conclusion}

Currently, with renewed interests in placebo phenomenon, it has been increasingly recognized that we have to renew our look at what might be non-specific and specific factors involved in the context of the clinical provision of acupuncture and that acupuncture as a 'whole system' may be much more than the process of penetrating needling $(47,56)$. These new ideas predict that research focus of acupuncture is shifting from proving specific efficacy of acupuncture to exploring how contextual factors are engaged to work, the association between needle insertion and the psychosocial context in which it is administered, and the association between acupuncture and placebo.

It has been suggested that both specific and nonspecific factors may play a role in the effectiveness of acupuncture $(10,49)$. The present hypothesis furthers this assumption in proposing that needle insertion does not make direct impacts that are independent of the underlying contextual factors but rather makes effects through the therapeutic context in which it is administrated. In other words, needle insertion into specific sites on the body acts on expectation mechanisms via attentional mechanisms, thus working through potentiation and modulation of expectation activated opioid systems. Clinical practice and theory basis of acupuncture can deserve to be a source of insight into endogenous opioid mechanisms about which still little is known. Expectation activated endogenous opioid systems can be potentiated and modulated to work in different patterns, different needle manipulations applied in acupoints located along different meridians corresponding respectively to these different patterns of modulation of expectation activated endogenous opioids. It is worth noting that this hypothesis does not preclude possible involvement of other mechanisms in the effectiveness of acupuncture. For example, conditioning mechanisms may present in repeated administration of acupuncture (57).

The present hypothesis derives mainly from TCM theory basis of acupuncture, close observation of daily practice of acupuncture, and limited evidence from acupuncture literature and research on the placebo effect. Though not giving answers to all the questions concerning acupuncture, this 'holistic' approach to the role of needle insertion provides a basis for further inquiry into the mode of action of acupuncture. Acupuncture as a therapy demonstrates how endogenous opioids can be potentiated and modulated through different needle manipulations applied in specific points on the body, configuring an internal homeostatic process that transforms enhanced expectations and beliefs into a healing power. It should also be recognized that this proposed mode of action of acupuncture is not simply acupuncture oriented; reversibility would give researchers a lead in unraveling the complex mechanisms of the placebo response. Though considerable progress has been made in our understanding of the neurobiological mechanisms of the placebo effect, research in this area is still in its infancy and most of our knowledge originates from the field of pain and analgesia (29). Given that acupuncture is widely used in more than pain conditions, its theory basis and daily practice will broaden our view of the placebo effect and allow us to better understand complex mind-brain-body interactions. Neurophysiological and neuroimaging studies on 
acupuncture will promise to cast direct light on the neurobiological mechanisms that are involved in acupuncture, and beyond acupuncture, the mechanisms of complex placebo phenomenon, not only for pain but also for other conditions.

\section{References}

1. Kaptchuk TJ. Acupuncture: theory, efficacy, and practice. Ann Intern Med 2002;136:374-83.

2. Liu T, Liu C. Acupuncture for treating osteoarthritis of the knee and hip. Arthritis Rheum 2006;54:3375-7.

3. Tan S, Tillisch K, Mayer E. Functional somatic syndromes: emerging biomedical models and traditional Chinese medicine. Evid Based Complement Alternat Med 2004;1:35-40.

4. Moerman DE, Jonas WB. Deconstructing the placebo effect and finding the meaning response. Ann Intern Med 2002;136:471-6.

5. Liu T. Role of acupuncturists in acupuncture treatment. Evid Based Complement Alternat Med 2007;4:3-6.

6. Bausell RB, Lao L, Bergman S, Lee WL, Berman BM. Is acupuncture analgesia an expectancy effect?: preliminary evidence based on participants' perceived assignments in two placebocontrolled trials. Eval Health Prof 2005;28:9-26.

7. Geers AL, Helfer SG, Weiland PE, Kosbab K. Expectations and placebo response: a laboratory investigation into the role of somatic focus. J Behav Med 2006;29:171-8.

8. MacPherson H, Thomas K, Walters S, Fitter M. The York acupuncture safety study: prospective survey of 34000 treatments by traditional acupuncturists. BMJ 2001;323:486-7.

9. Cho ZH, Chung SC, Jones JP, Park JB, Park JH, Lee HJ, Wong EK, Min BI. New findings of the correlation between acupoints and corresponding brain cortices using functional MRI. Proc Natl Acad Sci USA 1998:95:2670-3.

10. Pariente J, White P, Frackowiak RSJ, Lewith G. Expectancy and belief modulate the neuronal substrates of pain treated by acupuncture. Neuroimage 2005;25:1161-7.

11. Kong J, Gollub RL, Rosman IS, Webb JM, Vangel MG, Kirsch I, Kaptchuk TJ. Brain activity associated with expectancy-enhanced placebo analgesia as measured by functional magnetic resonance imaging. $J$ Neurosci 2006;26:381-8.

12. Vickers A, Wilson P, Kleijnen J. Acupuncture. Qual Saf Health Care 2002:11:92-7.

13. Ma SX. Neurobiology of acupuncture: toward CAM. Evid Based Complement Alternat Med 2004;1:41-7.

14. Lewith GT, White PJ, Pariente J. Investigating acupuncture using brain imaging techniques: the current state of play. Evid Based Complement Alternat Med 2005;2:315-9.

15. Ernst E. Acupuncture-a critical analysis. $J$ Intern Med 2006;259:125-37.

16. Lewith GT, White PJ, Kaptchuk TJ. Developing a research strategy for acupuncture. Clin J Pain 2006;22:632-8.

17. Joyce CRB. Placebo and complementary medicine. Lancet 1994;334:1279-81.

18. Vallance AK. Something out of nothing: the placebo effect. Advan Psychiatr Treat 2006;12:287-96.

19. Benedetti F, Mayberg HS, Wager TD, Stohler CS, Zubieta JK. Neurobiological mechanisms of the placebo effect. $J$ Neurosci 2005;25:10390-402.

20. Wager TD, Rilling JK, Smith EE, Sokolik A, Casey KL, Davidson RJ, Kosslyn SM, Rose RM, Cohen JD. Placebo-induced changes in FMRI in the anticipation and experience of pain. Science 2004;303:1162-7.

21. Petrovic P, Kalso E, Petersson KM, Ingvar M. Placebo and opioid analgesia-imaging a shared neuronal network. Science 2002;295:1737-40.

22. Benedetti F, Amanzio M. The neurobiology of placebo: from endogenous opioids to cholecystokinin. Prog Neurobiol 1997;52:109-25.

23. Benedetti F. The opposite effects of the opiate antagonist naloxone and the cholecystokinin antagonist proglumide on placebo analgesia. Pain 1996;64:535-43.
24. Amanzio M, Benedetti F. Neuropharmacological dissection of placebo analgesia: expectation-activated opioid systems versus conditioning-activated specific subsystems. J Neurosci 1999;19: 484-94.

25. Mayer E J, Price DD, Rafii A. Antagonism of acupuncture analgesia in man by the narcotic antogonist naloxone. Brain Res 1977; 121:368-72.

26. Cheng RSS, Pomeranz BH. Electroacupuncture analgesia is mediated by stereospecific opiate receptors and is reversed by antagonists of type 1 receptors. Life Sci 1980;26:631-8.

27. Sjolund BH, Eriksson MB. The influence of naloxone on analgesia produced by peripheral conditioning stimulation. Brain Res 1979;173:295-302.

28. Han JS, Ding XZ, Fan SG. Cholecystokinin octapeptide (CCK-8): Antagonism to electroacupuncture analgesia and a possible role inelectroacupuncture tolerance. Pain 1986;27:101-15.

29. Colloca L, Benedetti F. Placebos and painkillers: is mind as real as matter? Nature Rev Neurosci 2005;6:545-52.

30. Wager TD, Scott DJ, Zubieta JK. Placebo effects on human $\mu$-opioid activity during pain. PNAS 2007;104:11056-61.

31. Benedetti F, Pollo A, Lopiano L, Lanotte M, Vighetti S, Rainero I. Conscious expectation and unconscious conditioning in analgesic, motor, and hormonal placebo/nocebo responses. $J$ Neurosci 2003;23:4315-23.

32. Stewart-Williams S, Podd J. The placebo effect: dissolving the expectancy versus conditioning debate. Psychol Bull 2004:130:324-40

33. Pollo A, Amanzio M, Arslanian A, Casadio C, Maggi G, Benedetti F. Response expectancies in placebo analgesia and their clinical relevance. Pain 2001;93:77-84.

34. Voudouris NJ, Peck CL, Coleman G. The role of conditioning and expectancy in the placebo response. Pain 1990;43:121-8.

35. Walach H, Schmidt S, Dirhold T, Nosch S. The effects of a caffeineplacebo and suggestion on blood pressure, heart rate, well-being, and cognitive performance. Int. J. Psychophysiol 2002;43:247-460.

36. Montgomery GH, Kirsch I. Mechanisms of placebo pain reduction: an empirical investigation. Psychol Sci 1996;7:174-6.

37. Benedetti F, Arduino C, Amanzio M. Somatotopic activation of opioid systems by target-directed expectations of analgesia. J Neurosci 1999;9:3639-48.

38. WU MT, Hsieh JC, Xiong J, Yang CF, Pan HB, Chen YI, Tsai G, Rosen BR, Kwong KK. Central nervous pathway for acupuncture stimulation: localization of processing with functional MR Imaging of the brain-preliminary experience. Neuroradiol 1999;212:133-41.

39. Ernst E. Towards a scientific understanding of placebo effects. In: Peters D (ed). Understanding the Placebo Effect in Complementary Medicine. Theory, Practice and Research. London: ChurchillLivingstone, 2001, 17-30.

40. Harrington A. The Placebo Effect: An Interdisciplinary Phenomenon. Cambridge: Harvard University Press, 1999.

41. Crow R, Gage H, Hampson S, Hart J, Kimber A, Thomas H. The role of expectancies in the placebo effect and their use in the delivery of health care. Health Technol Assessm 1999;3:1-96.

42. Geers AL, Helfer SG, Kosbab K, Weiland PE, Landry SJ. Reconsidering the role of personality in placebo effects: dispositional optimism, situational expectations, and the placebo response. $J$ Psychosomat Res 2005;58:121-7.

43. Jospe M. The Placebo Effect in Healing. Lexington, MA: Health, 1978.

44. Kaptchuk TJ. The Placebo effect in alternative medicine: can the performance of a healing ritual have clinical significance? Ann Intern Med 2002;136:817-25.

45. Medvedev VI, Zavyalova EK, Ovchinnikov BV, Posokhova ST. Functional structure of the placebo response. Hum Physiol 1984;10:216-21.

46. Shapiro AK, Mike V, Barten H, Shapiro E. Study of the placebo effect with a self-administered placebo test. Compr Psychiatry 1973;14:535-48.

47. Paterson C, Dieppe P. Characteristic and incidental (placebo) effects in complex interventions such as acupuncture. Brit Med J 2005;330:1202-5. 
48. Dhond R, Kettner N, Napadow V. Neuroimaging acupuncture effects in the human brain. $J$ Alternat Complement Med 2007;13:603-16.

49. Dhond R, Kettner N, Napadow V. Do the neural correlates of acupuncture and placebo effects differ? Pain 2007;128:8-12.

50. Smith EE, Jonides J. Storage and executive processes in the frontallobes. Science 1999;283:1657-61.

51. Kaptchuk TJ. Powerful placebo: the dark side of the randomized controlled trial. Lancet 1998;351:1722-5.

52. Boutron I, Tubach F, Giraudeau B, Ravaud P. Methodological differences in clinical trials evaluating nonpharmacological and pharmacological treatments of hip and knee osteoarthritis. JAMA 2003;290:1062-70.

53. Kaptchuk TJ. The double-blind, randomized, placebo-controlled trial: gold standard or golden calf? J Clin Epidemiol 2001;54:541-9.
54. van Weel C. Examination of context of medicine. Lancet 2001;357:733-4.

55. Assefi NP, Sherman KJ, Jacobsen C, Goldberg J, Smith WR, Buchwald D. A randomized clinical trial of acupuncture compared with sham acupuncture in fibromyalgia. Ann Intern Med 2005;143:10-9

56. Verhoef M, Lewith $\mathrm{G}$, Ritenbaugh $\mathrm{C}$, Thomas $\mathrm{K}$, Boon $\mathrm{H}$, Fonnebo V. Whole systems research: moving forward. Focus Altern Complement Ther 2004;9:87-90.

57. Pomeranz B, Warma N. Electroacupuncture suppression of a nociceptive reflex is potentiated by two repeated electroacupuncture treatments: the first opioid effect potentiates a second non-opioid effect. Brain Res 1988;452:232-6.

Received July 18, 2007; accepted December 17, 2007 


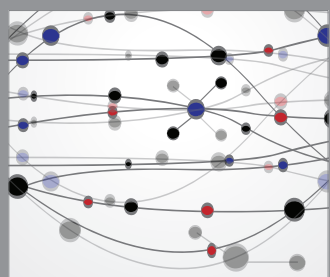

The Scientific World Journal
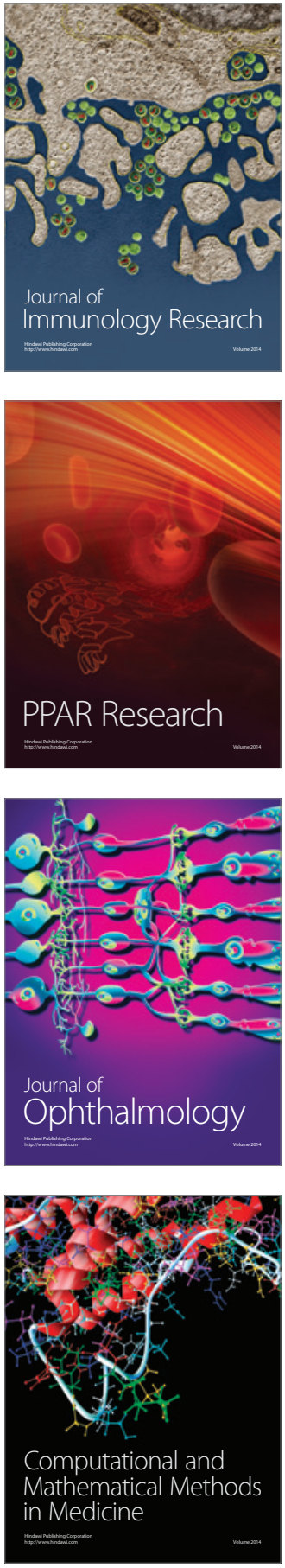

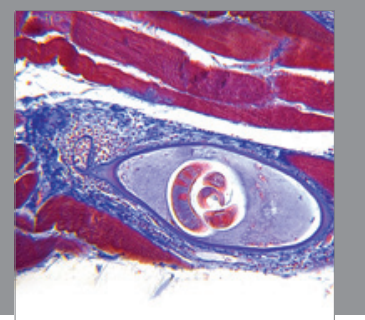

Gastroenterology

Research and Practice
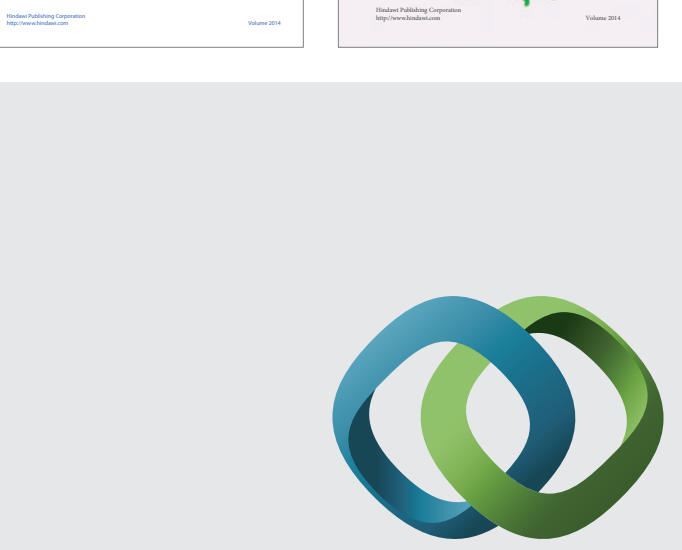

\section{Hindawi}

Submit your manuscripts at

http://www.hindawi.com
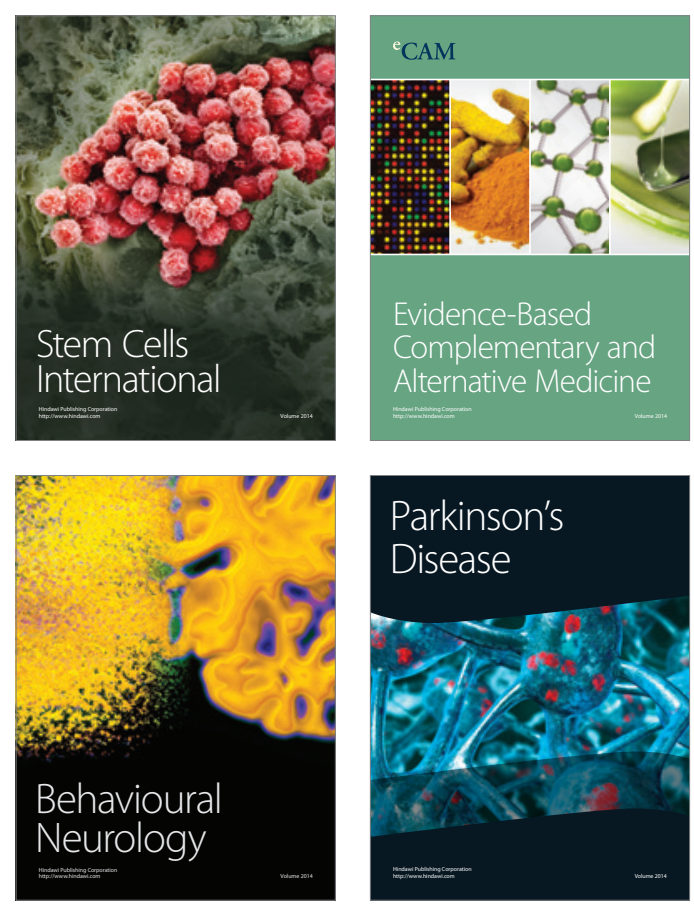

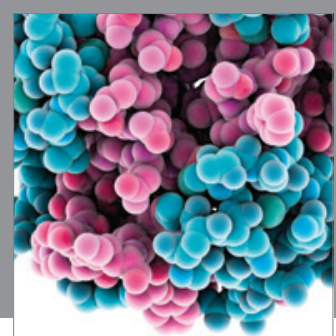

Journal of
Diabetes Research

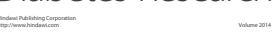

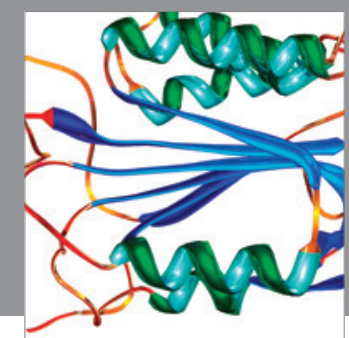

Disease Markers
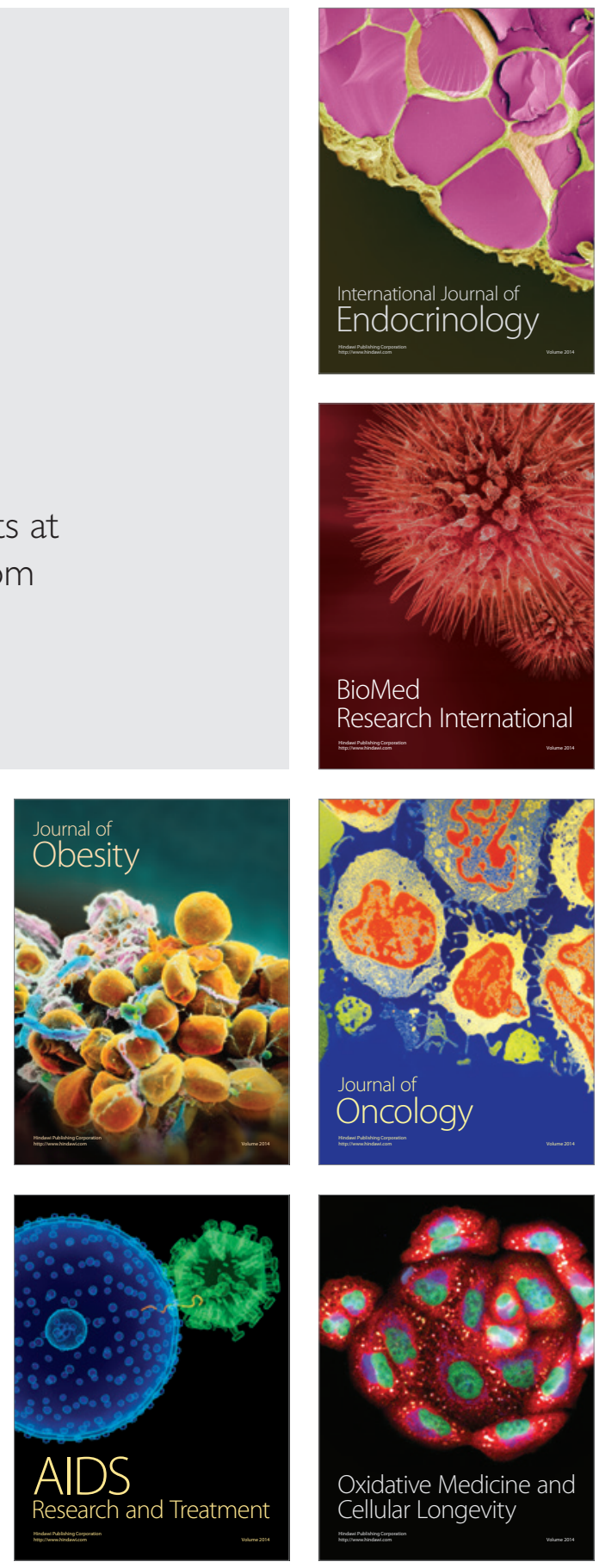